\title{
UNIQUENESS AND EXAMPLES OF COMPACT TORIC SASAKI-EINSTEIN METRICS
}

\author{
KOJI CHO, AKITO FUTAKI, AND HAJIME ONO
}

\begin{abstract}
In [1] it was proved that, given a compact toric Sasaki manifold with positive basic first Chern class and trivial first Chern class of the contact bundle, one can find a deformed Sasaki structure on which a SasakiEinstein metric exists. In the present paper we first prove the uniqueness of such Einstein metrics on compact toric Sasaki manifolds modulo the action of the identity component of the automorphism group for the transverse holomorphic structure, and secondly remark that the result of [11 implies the existence of compatible Einstein metrics on all compact Sasaki manifolds obtained from the toric diagrams with any height, or equivalently on all compact toric Sasaki manifolds whose cones have flat canonical bundle. We further show that there exists an infinite family of inequivalent toric Sasaki-Einstein metrics on $S^{5} \sharp k\left(S^{2} \times S^{3}\right)$ for each positive integer $k$.
\end{abstract}

\section{INTRODUCTION}

In 11 the existence of an Einstein metric is proved on a compact toric Sasaki manifold with positive basic first Chern class and trivial first Chern class of the contact bundle $D$; These two conditions will be denoted by $c_{1}^{B}>0$ and $c_{1}(D)=0$. The purposes of the present paper is firstly to prove the uniqueness of SasakiEinstein metrics up to a connected Lie group action and secondly to clarify the meaning of the assumptions $c_{1}^{B}>$ and $c_{1}(D)=0$ in relation with toric diagrams.

A Sasaki manifold is a Riemannian manifold $(S, g)$ whose cone manifold $(C(S), \bar{g})$ with $C(S) \cong S \times \mathbb{R}^{+}$and $\bar{g}=d r^{2}+r^{2} g$ is Kähler where $r$ is the standard coordinate on $\mathbb{R}^{+}$. From this definition $S$ is odd-dimensional and we put $\operatorname{dim} S=2 m+1$, and thus $\operatorname{dim}_{C(S)}=m+1$. A Sasaki manifold $(S, g)$ is said to be toric if the Kähler cone manifold $C(S)$ is toric, namely $(m+1)$-dimensional torus $G$ acts on $(C(S), \bar{g})$ effectively as holomorphic isometries. Note that $C(S)$ does not contain the apex. Then $S$ is a contact manifold with the contact form

$$
\eta=\left.(i(\bar{\partial}-\partial) \log r)\right|_{r=1}
$$

where $S$ is identified with the submanifold $\{r=1\} \subset C(S)$, and has the Reeb vector field $\xi$ with the defining properties

$$
i(\xi) \eta=1 \quad \text { and } \quad i(\xi) d \eta=0
$$

where $i(\xi)$ denotes the inner product. The Reeb field $\xi$ is a Killing vector field on $S$ and also lifts to a Killing vector field on $C(S)$, and thus $\xi$ is contained in the Lie algebra $\mathfrak{g}$ of $G$ since $G$ already has the maximal dimension of possible torus actions on $C(S)$.

Date: November 13, 2006 .

1991 Mathematics Subject Classification. Primary 53C55, Secondary 53C21, 55N91 .

Key words and phrases. Sasaki manifold, Einstein metric, toric diagram. 
The Reeb vector field $\xi$ generates a 1-dimensional foliation, called the Reeb foliation, on $S$. Since $\xi$ naturally lifts to a holomorphic vector field on $C(S)$ in the form $\xi-i J \xi$ with $\xi=J\left(r \frac{\partial}{\partial r}\right)$ the Reeb foliation shares common local leaf spaces with the holomorphic flow generated by $\xi-i J \xi$ on $C(S)$. Thus the local leaf spaces give the Reeb foliation a transverse holomorphic structure. The contact structure of $S$ determines a Kähler structure on the transverse holomorphic structure, which we call the transverse Kähler structure.

Recall that a smooth differential form $\alpha$ on $S$ is basic if

$$
i(\xi) \alpha=0 \quad \text { and } \quad \mathcal{L}_{\xi} \alpha=0
$$

where $\mathcal{L}_{\xi}$ denotes the Lie derivative by $\xi$. The basic forms are preserved by the exterior derivative $d$ which decomposes into $d=\partial_{B}+\bar{\partial}_{B}$, and we can define basic cohomology groups and basic Dolbealt cohomology groups. We also have the transverse Chern-Weil theory and can define basic Chern classes for complex vector bundles with basic transition functions. The Sasaki manifold is said to have positive basic first Chern class if the first Chern class of the normal bundle of the Reeb foliation is represented by a positive basic $(1,1)$-form; as mentioned above this condition is denoted by $c_{1}^{B}>0$. This is a necessary condition for the existence of Sasaki-Einstein metric or equivalently the existence of positive transverse Kähler-Einstein metric. There is another necessary condition $c_{1}(D)=0$ as a de Rham cohomology class where $D=\operatorname{Ker} \eta$ is the toric bundle. Coversely if $c_{1}^{B}>0$ and $c_{1}(D)=0$ then $c_{1}^{B}=\tau[d \eta]$ for some positive constant $\tau$. See Proposition 4.3 in 11] for more details. Given a Sasaki manifold $(S, g)$, we say that another Sasaki metric $g^{\prime}$ is compatible with the Sasaki structure of $(S, g)$ if $g$ and $g^{\prime}$ have the same Reeb vector field and thus define the same transverse holomorphic structure.

The automorphism group of the the transverse holomorphic structure is the group of all biholomorphic automorphisms of $C(S)$ which commute with the holomorphic flow generated by $\xi-i J \xi$. Such automorphisms descend to an action on $S$ preserving the transverse holomorphic holomorphic structure of the Reeb foliation, see section 2 for more detail. In this paper we first prove the uniqueness theorem of compatible Sasaki-Einstein metrics modulo connected group actions of automorphisms for the transverse holomorphic structure.

Theorem 1.1. Let $(S, g)$ be a compact toric Sasaki manifold with $c_{1}^{B}>0$ and $c_{1}(D)=0$. Then the identity component of the automorphism group for the transverse holomorphic structure acts transitively on the space of all Sasaki-Einstein metrics compatible with $g$.

In order to make clear which Sasaki manifolds the result of [1] applies to, we wish to explain the conditions $c_{1}^{B}>0$ and $c_{1}(D)=0$. Since a three dimensional Einstein manifold of positive scalar curvature is finitely covered by the standard three sphere we may restrict ourselves to the case when the dimension of $S$ is bigger than or equal to five.

Theorem 1.2. Let $S$ be a compact toric Sasaki manifold with $\operatorname{dim} S \geq 5$. Then the following three conditions are equivalent.

(a) $c_{1}^{B}>0$ and $c_{1}(D)=0$.

(b) The Sasaki manifold $S$ is obtained from a toric diagram with height $\ell$ for some positive integer $\ell$ defined by $\lambda_{1}, \cdots, \lambda_{d} \in \mathfrak{g}$ and $\gamma \in \mathfrak{g}^{*}$ (c.f. Definition 
3.1 and 3.2) and the Reeb field $\xi \in \mathfrak{g}$ satisfies

$$
\langle\gamma, \xi\rangle=-m-1 \text { and }\langle y, \xi\rangle>0 \text { for all } y \in C
$$

where $C=\left\{y \in \mathfrak{g}^{*} \mid\left\langle y, \lambda_{j}\right\rangle \geq 0, j=1, \cdots, d\right\}$.

(c) For some positive integer $\ell$, the $\ell$-th power $K_{C(S)}^{\otimes \ell}$ of the canonical line bundle $K_{C(S)}$ is trivial.

Remark 1.3. We denote by $\overline{C(S)}$ the the closure of the cone $C(S)$, that is $C(S)$ plus the apex, and consider it as an affine toric variety. It is a known fact that the condition of toric diagram with height $\ell$ is equivalent to the apex being a $\mathbb{Q}$ Gorenstein singularity, that is the $\ell$-th power $\mathcal{K}_{\overline{C(S)}}^{\otimes \ell}$ of the canonical sheaf $\mathcal{K}_{\overline{C(S)}}$ is invertible, see [2].

In the literature there are toric Sasaki manifolds denoted by $Y^{p, q}([12],[19)$, $L^{p, q, r}$ ([9], [19]), $X^{p, q}([14])$ and $Z^{p, q}([24]$, 3]) which are constructed from toric diagrams with height 1 . They are all of positive basic first Chern class by Theorem 1.2. and thus admit a Sasaki-Einstein metric by [11. Combining the existence result of 11 with Theorem 1.1 and Theorem 1.2 we get the following corollary.

Corollary 1.4. Given a toric diagram, there is a unique Sasaki structure whose cone is the one obtained from the toric diagram by Delzant construction and on which there exist compatible Einstein metrics. Moreover the identity component of the automorphism group of the transverse holomorphic structure acts transitively on the set of all compatible Einstein metrics.

Thus the Sasaki-Einstein metrics constructed in [11] on $Y^{p, q}$ coincide with those which have been known in the literature [12, [19].

Using diagrams we show that compact connected toric Sasaki manifolds associated with toric diagrams of height bigger than 1 are not simply connected and that the converse is not true by giving an example. We will also show the following.

Theorem 1.5. For each positive integer $k$ there exists an infinite family of inequivalent toric Sasaki-Einstein metrics on the $k$-fold connected sum $S^{5} \sharp k\left(S^{2} \times S^{3}\right)$ of $S^{2} \times S^{3}$ with $S^{5}$.

The existence of (possibly non-toric) Sasaki-Einstein metrics on $S^{5} \sharp k\left(S^{2} \times S^{3}\right)$ has been known by the works of Boyer, Galicki, Nakamaye and Kollár ([5, 4, [15]), and that the existence of toric Sasaki-Einstein metrics for all odd $k$ 's has been known by van Coevering ([26]). Hence our result is new in that we obtain toric constructions for all even $k$ 's. Moreover most of our examples should be irregular while the previous ones are all quasi-regular.

We are grateful to Charles Boyer for pointing out our careless statement of the results without the condition $c_{1}(D)=0$ in the first version of the paper.

\section{Uniqueness of compatible SASAKi-Einstein metrics}

In Kähler geometry a well known method of proving uniqueness of constant scalar curvature metrics is to use geodesics on the space of all Kähler metrics in a fixed Kähler class ([18, [10, [8]). This idea becomes substantially simpler when the Kähler manifold under consideration is toric because the geodesic becomes a line segment expressed by the symplectic potentials (13]). To prove Theorem 1.1 we wish to use the same idea, but have to consider geodesics both on the space of 
transverse Kähler metrics on $S$ and on the space of Kähler metrics on $C(S)$. We therefore give an outline of the idea in the case of compact Kähler manifolds first and then explain how we modify it in the Sasakian case.

Let $V$ be a compact Kähler manifold and $\mathcal{H}$ the space of Kähler potentials in a fixed Kähler class $\left[\omega_{0}\right]$ :

$$
\mathcal{H}=\left\{\varphi \in C^{\infty}(V) \mid \omega_{\varphi}=\omega_{0}+\sqrt{-1} \partial \bar{\partial} \varphi>0\right\} .
$$

The tangent space $T_{\varphi} \mathcal{H}$ at $\varphi \in \mathcal{H}$ is identified with the set $C^{\infty}(V)$ of all real smooth functions via

$$
\frac{d}{d s} \mid s=0_{s}(\varphi+s \psi)=\psi \in C^{\infty}(V) .
$$

We have a natural Riemannian metric on $\mathcal{H}$

$$
\left(\psi_{1}, \psi_{2}\right):=\int_{M} \psi_{1} \psi_{2} \omega_{\varphi}^{n}
$$

where $n=\operatorname{dim}_{\mathbb{C}} V$ and $\psi_{1}, \psi_{2} \in T_{\varphi} \mathcal{H} \cong C^{\infty}(V)$. For a smooth path $\varphi=\left\{\varphi_{t} \mid a \leq\right.$ $t \leq b\}$ in $\mathcal{H}$, let $\psi=\left\{\psi_{t} \mid a \leq t \leq b\right\}$ be a vector field along $\varphi$, considered as

$$
\psi_{t}=\frac{d}{d s \mid s=0}\left(\varphi_{t}+s \psi_{t}\right) \in T_{\varphi_{t}} \mathcal{H}, \quad a \leq t \leq b .
$$

Then the covariant derivative by Levi-Civita connection is expressed as

$$
\frac{D}{\partial t} \psi=\dot{\psi}_{t}-\operatorname{Re}\left(\bar{\partial} \dot{\varphi}_{t}, \bar{\partial} \psi_{t}\right)_{\omega_{t}}=\dot{\psi}_{t}-\frac{1}{2}\left(d \dot{\varphi}_{t}, d \psi_{t}\right)_{\omega_{t}}
$$

where $\omega_{t}=\omega_{\varphi_{t}}$. Thus the equation of geodesics is given by

$$
\ddot{\varphi}_{t}-\left|\bar{\partial} \dot{\varphi}_{t}\right|_{\omega_{t}}^{2}=0 .
$$

The K-energy, or Mabuchi energy, is defined by

$$
\mu\left(\omega_{\varphi}\right):=M\left(\omega_{\varphi}, \omega_{0}\right)=-\int_{0}^{1}\left(\int_{V}\left(\sigma_{\omega_{t}}-\bar{\sigma}\right) \dot{\varphi} \omega_{t}^{n}\right) d t
$$

where $\varphi_{t}:=t \varphi, 0 \leq t \leq 1, \omega_{t}=\omega_{\varphi_{t}}$ and

$$
\bar{\sigma}=\frac{\int_{V} n c_{1}(V) \omega_{0}^{n-1}}{\int_{V} \omega_{0}^{n}} .
$$

The fundamental facts are the following:

- $\omega_{\varphi}$ is a critical point of $\mu$ if and only if $\omega_{\varphi}$ is a Kähler metric of constant scalar curvature.

- The Hessian of $\mu$ is positive semi-definite, so $\mu$ is a convex function.

- We have for any smooth path $\left\{\varphi_{t}\right\}_{0 \leq t \leq 1}$

$$
\frac{d^{2} \mu\left(\omega_{t}\right)}{d t^{2}}=\int_{V}\left|\bar{\partial} Y_{t}\right|_{\omega_{t}}^{2} \omega_{t}^{n}-\int_{V}\left(\ddot{\varphi}_{t}-\left|\bar{\partial} \dot{\varphi}_{t}\right|_{\omega_{t}}^{2}\right) \omega_{t}^{n}
$$

where $Y_{t}=\omega_{t}^{-1}\left(\bar{\partial} \dot{\varphi}_{t}\right)$. In particular, for geodesics $\varphi_{t}$ we have

$$
\frac{d^{2}}{d t^{2}} \mu\left(\omega_{t}\right)=\int_{V}\left|\bar{\partial} Y_{t}\right|_{\omega_{t}}^{2} \omega_{t}^{n} \geq 0
$$


Now we can prove the uniqueness of constant scalar curvature metrics modulo the action of the identity component of the group of biholomorphic automorphisms of $V$ provided we have a geodesic joining two such metrics as follows. Suppose that both $\omega_{0}$ and $\omega_{1}$ are Kähler forms with constant scalar curvature and that we have a geodesic $\omega_{t}, 0 \leq t \leq 1$, joining them. Then it follows from the above facts that

$$
\frac{d^{2} \mu\left(\omega_{t}\right)}{d t^{2}} \geq 0, \quad{\frac{d \mu\left(\omega_{t}\right)}{d t}}_{\mid t=0}=0, \quad{\frac{d \mu\left(\omega_{t}\right)}{d t}}_{\mid t=1}=0 .
$$

These imply $\frac{d \mu\left(\omega_{t}\right)}{d t}=0$ for all $t \in[0,1]$. But the equation (3) shows that $Y_{t}$ is a holomorphic vector field and $\omega_{t}$ is the pull-back of $\omega_{0}$ by an automorphism of $V$.

The problem is then whether we can find a geodesic in the space of Kähler metrics. The geodesic equation is reduced to a degenerate Monge-Ampère equation ([25]). The existence of $C^{1,1}$-solution was proved by X.X. Chen [8], but $C^{1,1}$ geodesics are not enough to prove uniqueness and Chen used $\varepsilon$-approximations of the solutions. In the toric case, however, geodesics are obtained as a line segment of symplectic potentials as shown by Guan [13, which is explained next.

Let $V$ be a toric Kähler manifold. Then $V$ is a completion of $\left(\mathbb{C}^{*}\right)^{n}$ with coordinates $w^{1}, \cdots, w^{n}$. Put $w^{j}=e^{z^{j}}$ and $z^{j}=x^{j}+i \theta^{j}$. Let $F(x)$ be the Kähler potential of a $T^{n}$-invariant Kähler metric so that

$$
\omega=i g_{j} d_{z^{j}} \wedge d \bar{z}^{k}=\frac{i}{4} \frac{\partial^{2} F}{\partial x^{j} \partial x^{k}} d z^{j} \wedge d \bar{z}^{k} .
$$

The symplectic potential $G$ is the Legendre transform of $\mathrm{F}$ :

$$
G(y)=\sum_{j=1}^{n} x^{j} \frac{\partial F}{\partial x^{j}}-F
$$

with $y_{j}=\frac{\partial F}{\partial x^{j}}$. There is a symmetrical relation

$$
x^{j}=\frac{\partial G}{\partial y_{j}}, \quad F(x)=\sum_{j=1}^{n} y_{j} \frac{\partial G}{\partial y_{j}}-G .
$$

Thus as matrices

$$
\left(\frac{\partial^{2} F}{\partial x^{i} \partial x^{j}}\right)=\left(\frac{\partial y_{i}}{\partial x^{j}}\right)=\left(\frac{\partial x^{i}}{\partial y_{j}}\right)^{-1}=\left(\frac{\partial^{2} G}{\partial y_{i} \partial y_{j}}\right)^{-1} .
$$

If $\left\{\omega_{t}\right\}$ is a curve in the space of Kähler forms and $F_{t}$ is the corresponding Kähler potential then we have the $t$-dependent coordinates

$$
y_{t}=\frac{\partial F_{t}}{\partial x}
$$

on the image of the moment map. Conversely if we start from a curve $G_{t}$ of symplectic potential with $t$-independent coordinates $y$ we have $t$-dependent coordinates

$$
x_{t}=\frac{\partial G_{t}}{\partial y}
$$

on $\mathbb{R}^{n}$. To understand the geodesic equation better it is convenient to consider $F_{t}\left(x_{t}\right)$ in terms of $t$-dependent coordinates $x_{t}$ and $G_{t}\left(y_{t}\right)$ in terms of $y_{t}$ with the relations

$$
y_{t j}=\frac{\partial F_{t}}{\partial x_{t}^{j}}, \quad G_{t}\left(y_{t}\right)=\sum_{j=1}^{n} x_{t}^{j} \frac{\partial F_{t}}{\partial x_{t}^{j}}-F_{t} .
$$


We suppress $t$ for the notational convenience. First of all

$$
\frac{\partial G(y)}{\partial t}=\dot{G}(y)+\sum_{j=1}^{n} \frac{\partial G}{\partial y_{j}} \dot{y}_{j}=\dot{G}(y)+\sum_{j=1}^{n} x^{j} \dot{y}_{j},
$$

and

$$
\begin{aligned}
\frac{\partial}{\partial t}\left(\sum_{j=1}^{n} x^{j} \frac{\partial F}{\partial x^{j}}-F\right) & =\sum_{j=1}^{n}\left(\dot{x}^{j} \frac{\partial F}{\partial x^{j}}+x^{j} \frac{\partial \dot{F}}{\partial x^{j}}+x^{j} \frac{\partial^{2} F}{\partial x^{j} \partial x^{k}} \dot{x}^{k}-\frac{\partial F}{\partial x^{j}} \dot{x}^{j}\right)-\dot{F} \\
& =\sum_{j=1}^{n} x^{j} \dot{y}^{j}-\dot{F}(x) .
\end{aligned}
$$

Thus

$$
\dot{G}(y)=-\dot{F}(x) .
$$

Taking the derivative of (5) we get

$$
\ddot{F}+\sum_{j=1}^{n} \frac{\partial \dot{F}}{\partial x^{j}} \dot{x}^{j}=-\ddot{G}+\sum_{j=1}^{n} \frac{\partial \dot{G}}{\partial y^{j}} \dot{y}^{j} .
$$

In what follows we omit obvious indices and sum notations. Taking the derivative of $y=\frac{\partial F}{\partial x}$ we have

$$
\dot{y}=\frac{\partial \dot{F}}{\partial x}+\frac{\partial y}{\partial x} \dot{x} .
$$

Proposition 2.1. Let $M$ be a toric Kähler manifold.

(a) Let $F_{t}\left(x_{t}\right)$ and $G_{t}\left(y_{t}\right)$ be Kähler and symplectic potentials of $t$-dependent coordinates $x_{t}$ and $y_{t}$ satisfying the relations (4). Then the geodesic equations are insensitive to $t$-dependent coordinates in that

$$
\left(\ddot{F}-\frac{1}{2}|d \dot{F}|_{t}^{2}\right)\left(x_{t}\right)=0
$$

if and only if

$$
\ddot{G}\left(y_{t}\right)=0 .
$$

(b) For any two Kähler potential there exists a unique geodesic joining them. In the action-angle coordinates $y, \theta$ with fixed standard symplectic form $\omega=\sum_{i=1}^{n} d y_{i} \wedge d \theta^{i}$ the geodesic can be expressed as $t G_{1}(y)+(1-t) G_{0}(y)$.

Proof. (a) It follows from (6), (7) and (5) that

$$
\begin{aligned}
\left(\ddot{F}-\frac{1}{2}|d \dot{F}|_{t}^{2}\right)\left(x_{t}\right) & =\ddot{F}-\frac{\partial x}{\partial y} \frac{\partial \dot{F}}{\partial x} \frac{\partial \dot{F}}{\partial x} \\
& =-\ddot{G}-\frac{\partial \dot{G}}{\partial y} \dot{y}-\frac{\partial \dot{F}}{\partial x} \dot{x}-\frac{\partial x}{\partial y} \frac{\partial \dot{F}}{\partial x} \frac{\partial \dot{F}}{\partial x} \\
& =-\ddot{G}-\frac{\partial \dot{G}}{\partial y} \dot{y}-\frac{\partial \dot{F}}{\partial x} \dot{x}-\left(\frac{\partial x}{\partial y} \dot{y}-\dot{x}\right) \frac{\partial \dot{F}}{\partial x} \\
& =-\ddot{G}-\frac{\partial \dot{G}}{\partial y} \dot{y}-\frac{\partial \dot{F}}{\partial y} \dot{y} \\
& =-\ddot{G}\left(y_{t}\right) .
\end{aligned}
$$


(b) The existence of a geodesic joining two Kähler potentials can be shown as follows. We first fix $t$-independent coordinates $x$ on $\mathbb{R}^{n}$ and $y$ on the convex polytope. Let $F_{0}(x)$ and $F_{1}(x)$ be two Kähler potentials, and $y_{0}, y_{1}, G_{0}$ and $G_{1}$ be defined by

$$
y_{0}=\frac{\partial F_{0}}{\partial x}, G_{0}=x y_{0}-F_{0} ; \quad y_{1}=\frac{\partial F_{1}}{\partial x}, G_{1}=x y_{1}-F_{1} .
$$

Put $y_{t}=t y_{1}+(1-t) y_{0}$. Then $y_{t}$ is the moment map of the Kähler potential $t F_{1}+(1-t) F_{0}$ and thus gives coordinates on the image of the moment map. Put $G_{t}(y)=t G_{1}\left(y_{t}\right)+(1-t) G_{0}\left(y_{t}\right)$. Then obviously $\ddot{G}\left(y_{t}\right)=0$, so the corresponding Legendre transform

$$
F_{t}\left(x_{t}\right)=y_{t} \frac{\partial G_{t}}{\partial y_{t}}-G_{t}\left(y_{t}\right)
$$

satisfies the geodesic equation $\left.\left(\ddot{F}-\frac{1}{2}|d \dot{F}|_{t}^{2}\right)\right)\left(x_{t}\right)=0$. Thus we get a geodesic $F_{t}(x)$ joining $F_{0}$ and $F_{1}$. Notice that we inserted $t$-independent coordinates $x$ in $F_{t}$ so that $F_{t}(x)$ becomes a geodesic in the original sense. We could perturb $y_{t}$ and get a different $x_{t}$, but $F_{t}(x)$ does not change because of the uniqueness of the geodesic proved by X.-X. Chen [8]. One can argue as in [13] to show that $F_{t}$ defines metrics on the whole Kähler manifold.

Taking the Legendre transform of the geodesic $F_{t}$ obtained in this way one sees that in the action-angle coordinates on the polytope (see 1]) $G_{t}$ is expressed as $t G_{1}(y)+(1-t) G_{0}(y)$.

Now we consider the case of transverse Kähler structure of compact Sasaki manifolds of positive basic Chern class. We begin with the study of the automorphisms of transverse holomorphic structure.

Proposition 2.2. Let $S$ be a compact Sasaki manifold. Then the Lie algebra of the automorphism group of transverse holomorphic structure is the Lie algebra of all Hamiltonian holomorphic vector fields in the sense of Definition 4.4 of [11].

Proof. Since the Reeb foliation has transverse holomorphic structure we can choose local transverse holomorphic coordinates $z^{1}, \cdots, z^{m}$. They are used as part of local holomorphic coordinates as well as local coordinates on $S$. A local holomorphic vector field of the form $X^{i} \frac{\partial}{\partial z^{i}}$ is considered as a local vector field on $C(S)$ as well as one on $S$. We will denote by $\tilde{X}^{\prime}$ the former and by $X^{\prime}$ the latter. Note that, along $\{r=1\}, X^{\prime}$ is the tangential part to $S \cong\{r=1\}$ of $\widetilde{X}^{\prime}$.

If a vector field $\widetilde{X}$ generates a one-parameter group of automorphisms of $C(S)$ which commutes with the holomorphic flow generated by $\xi-i J \xi$ then $[\widetilde{X}, \xi-i J \xi]=$ 0 . If we set $\tilde{X}=Y-i J Y$ with $Y$ the real part of $\widetilde{X}$ then $[\xi, Y]=0$. From this one sees $[Y, J \xi]=J[Y, \xi]=0$. These mean that the holomorphic flow descends to $S$ and local leaf spaces, and that $\widetilde{X}$ descends to a holomorphic vector field on each local leaf space. This local vector field can be regarded as a local vector field $\widetilde{X}^{\prime}$ on $C(S)$ as well as $X^{\prime}$ on $S$. Recall from [11] that the contact form $\eta$ on $S$ lifts to $C(S)$ as

$$
\eta=2 d^{c} \log r=i(\bar{\partial}-\partial) \log r
$$

where we use the same letter $\eta$ by the abuse of notation. We then have $\eta\left(\tilde{X}^{\prime}\right)=$ $\eta\left(X^{\prime}\right)$. This is because if $p: C(S)=S \times \mathbb{R}_{+} \rightarrow S$ is the projection then $\eta$ on $C(S)$ is $p^{*}$ and $X^{\prime}=p_{*} \widetilde{X}^{\prime}$. 
Then $\widetilde{X}$ can be expressed as

$$
\widetilde{X}=\eta(\widetilde{X})(\xi-i J \xi)+\left(\tilde{X}^{\prime}-\eta\left(\tilde{X}^{\prime}\right)(\xi-i J \xi)\right) .
$$

Note that the right hand side is an orthogonal splitting. Taking $\bar{\partial}$ of both sides of (8) we get

$$
\bar{\partial} \eta(\widetilde{X})=\bar{\partial} \eta\left(\widetilde{X}^{\prime}\right)=\bar{\partial}_{B} \eta\left(X^{\prime}\right)
$$

Taking the tangential component to $S$ of $\widetilde{X}$ we obtain

$$
X:=\eta(\tilde{X}) \xi+X^{\prime}-\eta\left(X^{\prime}\right) \xi .
$$

Then since $\eta(X)=\eta(\widetilde{X}), X$ may be written as

$$
X=\eta(X) \xi+X^{\prime}-\eta\left(X^{\prime}\right) \xi
$$

Since $\eta$ is of the form

$$
\eta=d t-i \partial_{B} f+i \bar{\partial}_{B} f
$$

where $t$ is the leaf coordinate with $\xi t=1$ and $f$ is the Kähler potenitial for the transverse Kähler form $\frac{1}{2} d \eta$ we have

$$
d \eta=2 i \partial_{B} \bar{\partial}_{B} f .
$$

Hence we get

$$
i(X) d \eta=i\left(X^{\prime}\right) d \eta=2 i\left(X^{\prime}\right) \bar{\partial}_{B} \eta=-2 \bar{\partial}_{B}\left(\eta\left(X^{\prime}\right)\right)=-2 \bar{\partial}_{B}(\eta(X)) .
$$

Hence $X$ is a Hamiltonian holomorphic vector field in the sense of Definition 4.4 of 11. It is easy to see that the Lie algebra consisting of all $\widetilde{X}$ is isomorphic to the Lie algebra consisting of all Hamiltonian holomorphic vector fields $X$.

Recall that a basic function $\varphi$ is a smooth function on $S$ such that $\xi \varphi=0$ where $\xi$ is the Reeb field. The transverse Kähler form $\omega^{T}$ is given by

$$
\omega^{T}=\frac{1}{2} d \eta
$$

where

$$
\eta=\left.2 d^{c} \log r\right|_{\{r=1\} \cong S}=\left.i(\bar{\partial}-\partial) \log r\right|_{\{r=1\} \cong S}
$$

and the transverse Kähler deformation is given by $\omega^{T}+i \partial_{B} \bar{\partial}_{B} \varphi$ for some basic function $\varphi$ where $\partial_{B}$ and $\bar{\partial}_{B}$ are basic $\partial$ and $\bar{\partial}$-operators. The tangent space to a transverse Kähler metric is therefore the set of all basic functions $\varphi$. We may define geodesics in the space of transverse Kähler metrics by the equation

$$
\ddot{\varphi}-\left|\bar{\partial}_{B} \dot{\varphi}\right|_{t}^{2}=0 \text {. }
$$

We can derive the similar conclusion that if one can always find a geodesic joining two Kähler potentials one can show that the identity component of the automorphism group of the transverse holomorphic structure acts transitively on the space of transverse Kähler metrics of constant scalar curvature by using the principle stated in the Appendix of [11. In fact the corresponding equation to (31) shows that the geodesic joining two transverse Kähler metric of constant scalar curvature is tangent to the Hamiltonian function of a Hamiltonian holomorphic vector field. Of course since transverse Kähler-Einstein metrics have constant scalar curvature these arguments give the uniqueness of transverse Kähler-Einstein metrics modulo the action of the identity component of the automorphism group of the transverse holomorphic structure. 
Suppose now that the compact Sasaki manifold $S$ is toric so that the cone $C(S)$ is a toric Kähler manifold. We may also define the covariant derivative and geodesic equation by (11) and (2). By the above arguments we can always find a geodesic joining two Kähler potentials on $C(S)$. The Kähler form on $C(S)$ is given by

$$
\omega=\frac{1}{2} d\left(r^{2} \eta\right)=\frac{1}{2} i d d^{c} r^{2}=\frac{1}{2} i \partial \bar{\partial} r^{2} .
$$

A function on $S$ can be lifted to $C(S)=S \times \mathbb{R}^{+}$and we use the same notation for a function on $S$ and its lift to $C(S)$. The transverse Kähler deformation is given using a basic function $\varphi$ by

$$
\widetilde{\eta}=\eta+2 d_{B}^{c} \varphi=2 d^{c} \log (r \exp \varphi),
$$

and hence the Kähler form $\omega$ on $C(S)$ is deformed by

$$
\widetilde{\omega}=\frac{1}{2} d\left(\tilde{r}^{2} \widetilde{\eta}\right)=\frac{1}{2} i \partial \bar{\partial}\left(r^{2} \exp (2 \varphi)\right) .
$$

Let $\mathcal{K}$ be the space of all Kähler metrics on $C(S)$ of the form $i \partial \bar{\partial} H$ for some real smooth function $H$ on $C(S)$, and $\mathcal{K}_{\omega}$ be the submanifold consisting of Kähler metrics obtained by transverse Kähler deformations of the form (10).

Lemma 2.3. $\mathcal{K}_{\omega}$ is a totally geodesic submanifold in $\mathcal{K}$.

Proof. By (10) a curve in $\mathcal{K}_{\omega}$ is of the form $\frac{1}{2} r^{2} \exp \left(2 \varphi_{t}\right)$ so that its tangent vector is $r_{t}^{2} \dot{\varphi}_{t}$ where we put $r_{t}=r \exp \varphi_{t}$. Similarly a vector filed along $\frac{1}{2} r^{2} \exp \left(2 \varphi_{t}\right)$ is of the form $r_{t}^{2} \psi_{t}$ for a curve $\psi_{t}$ of basic functions. The covariant derivative of $r_{t}^{2} \psi_{t}$ along $\frac{1}{2} r^{2} \exp \left(2 \varphi_{t}\right)$ is computed by

$$
\begin{aligned}
\frac{D}{d t}\left(r_{t}^{2} \psi_{t}\right) & =2 r_{t}^{2} \dot{\varphi}_{t} \psi_{t}+r_{t}^{2} \dot{\psi}_{t}-\frac{1}{2}\left(d\left(r_{t}^{2} \dot{\varphi}_{t}\right), d\left(r_{t}^{2} \psi_{t}\right)\right) \\
& =r_{t}^{2}\left(\dot{\psi}_{t}-\frac{1}{2}\left(d \dot{\theta}_{t}, d \psi_{t}\right)\right) \\
& =r_{t}^{2} \frac{D}{d t} \psi_{t}
\end{aligned}
$$

where the covariant derivative in the last term is the one for the transverse Kähler structure. This shows that the covariant derivative of a vector field in the tangent spaces of $\mathcal{K}_{\omega}$ along a curve in $\mathcal{K}_{\omega}$ is tangent to $\mathcal{K}_{\omega}$. Thus $\mathcal{K}_{\omega}$ is a totally geodesic submanifold.

Proposition 2.4. A curve $i \partial \bar{\partial}\left(\frac{1}{2} r^{2} \exp \left(2 \varphi_{t}\right)\right)$ in $\mathcal{K}_{\omega}$ is a geodesic if and only if $\omega^{T}+i \partial_{B} \bar{\partial}_{B} \varphi_{t}$ is a geodesic in the space of the transverse Kähler metrics. Moreover, for any given two transverse Kähler metrics with the same Reeb field $\xi$ corresponding to toric Kähler cone metrics there exists a unique geodesic joining them.

Proof. The first statement follows immediately from (11). Let $\omega_{0}^{T}$ and $\omega_{1}^{T}$ be the transverse Kähler metrics with the common Reeb field $\xi$ corresponding to toric Kähler metrics $\omega_{0}$ and $\omega_{1}$ on $C(S)$. Let $G_{0}$ and $G_{1}$ be the corresponding symplectic potentials. We use the action-angle coordinates $y_{i}, \theta^{i}$. Since $G_{0}$ and $G_{1}$ have common Reeb field $\xi, g=G_{1}-G_{0}$ satisfies

$$
\left(\sum_{j=1}^{m+1} y_{j} \frac{\partial}{\partial y_{j}}\right) \frac{\partial g}{\partial y_{i}}=0
$$


by (2.40) in 21. Thus the geodesic $t G_{1}(y)+(1-t) G_{0}(y)=G_{0}+t g$ joining $G_{0}$ and $G_{1}$ has the same Reeb field $\xi$, and thus the corresponding Kähler potentials define the same transverse holomorphic structure. From the first statement it follows that the geodesic $F_{t}=\frac{1}{2} r_{t}^{2}=\frac{1}{2} r^{2} \exp \left(2 \varphi_{t}\right)$ with $\varphi_{t}$ basic smooth functions descends to a geodesic in the space of transverse Kähler metrics.

Proof of Theorem (1.1): Let $\omega_{0}^{T}$ and $\omega_{1}^{T}$ be the transverse Kähler metrics corresponding to two Sasaki-Einstein metrics. As proved in [22] and 7] LichnerowiczMatsushima theorem for compact Kähler manifolds of constant scalar curvature extends to compact Sasaki manifolds of constant transverse scalar curvature we may assume that both $\omega_{0}^{T}$ and $\omega_{1}^{T}$ are invariant under the maximal compact subgroup of the group of automorphisms of the transverse holomorphic structure. In particular we may assume that they are invariant under the maximal torus $G$, and thus we only need to consider the toric Sasaki-Eisntein metrics.

In [21] and [11] it is shown that the volume functional of Sasakian structures depends only on the Reeb fields, that there is a unique critical Reeb field $\xi$ which minimizes the volume functional and that only for the critical point $\xi$ the obstruction to the existence of transverse Kähler-Einstein metric vanishes. Thus $\omega_{0}^{T}$ and $\omega_{1}^{T}$ must have a common Reeb field $\xi$. They can be joined by a geodesic by Proposition 2.4. We then apply the standard method known in Kähler geometry as explained above, and it follows from (3) that the geodesic is tangent to the Hamiltonian function of a Hamiltonian holomorphic vector field. This completes the proof.

\section{TORIC DIAGRAMS}

We begin with the definition of a good rational polyhedral cone.

Definition 3.1 (c.f. [16]). Let $\mathfrak{g}^{*}$ be the dual of the Lie algebra $\mathfrak{g}$ of the $(m+1)$ dimensional torus $G$. Let $\mathbb{Z}_{\mathfrak{g}}$ be the integral lattice of $\mathfrak{g}$, that is the kernel of the exponential map exp : $\mathfrak{g} \rightarrow G$. A subset $C \subset \mathfrak{g}^{*}$ is a rational polyhedral cone if there exists a finite set of vectors $\lambda_{i} \in \mathbb{Z}_{\mathfrak{g}}, 1 \leq i \leq d$, such that

$$
C=\left\{y \in \mathfrak{g}^{*} \mid\left\langle y, \lambda_{i}\right\rangle \geq 0 \text { for } i=1, \cdots, d\right\} .
$$

We assume that the set $\lambda_{i}$ is minimal in that for any $j$

$$
C \neq\left\{y \in \mathfrak{g}^{*} \mid\left\langle y, \lambda_{i}\right\rangle \geq 0 \text { for all } i \neq j\right\}
$$

and that each $\lambda_{i}$ is primitive, i.e. $\lambda_{i}$ is not of the form $\lambda_{i}=a \mu$ for an integer $a \geq 2$ and $\mu \in \mathbb{Z}_{\mathfrak{g}}$. (Thus $d$ is the number of codimension 1 faces if $C$ has nonempty interior.) Under these two assumptions a rational polyhedral cone $C$ with nonempty interior is good if the following condition holds. If

$$
\left\{y \in C \mid\left\langle y, \lambda_{i_{j}}\right\rangle=0 \text { for all } j=1, \cdots, k\right\}
$$

is a non-empty face of $C$ for some $\left\{i_{1}, \cdots, i_{k}\right\} \subset\{1, \cdots, d\}$, then $\lambda_{i_{1}}, \cdots, \lambda_{i_{k}}$ are linearly independent over $\mathbb{Z}$ and

$$
\left\{\sum_{j=1}^{k} a_{j} \lambda_{i_{j}} \mid a_{j} \in \mathbb{R}\right\} \cap \mathbb{Z}_{\mathfrak{g}}=\left\{\sum_{j=1}^{k} m_{j} \lambda_{i_{j}} \mid m_{j} \in \mathbb{Z}\right\} .
$$

Let $M$ be a $2 m+1$-dimensional compact connected contact toric manifold with the contact form $\eta$. Namely there is an effective action of the $(m+1)$-dimensional torus $G$ which preserves $\eta$. Then the moment map $\mu: M \rightarrow \mathfrak{g}^{*}$ is defined by

$$
\langle\mu(p), X\rangle=\left(\eta\left(X_{M}\right)\right)(p)
$$


where $X_{M}$ denotes the the vector filed on $M$ induced by $X \in \mathfrak{g}$. We assume $\operatorname{dim} M=2 m+1 \geq 5$. It is well-known ([16]) that if the action of $G$ is not free then the image of the moment map is a good rational polyhedral cone.

Definition 3.2. An $(m+1)$-dimensional toric diagram with height $\ell$ is a collection of $\lambda_{i} \in \mathbb{Z}^{m+1} \cong \mathbb{Z}_{\mathfrak{g}}$ satisfying (12) and $\gamma \in \mathbb{Q}^{m+1} \cong\left(\mathbb{Q}_{\mathfrak{g}}\right)^{*}$ such that

(1) $\ell$ is a positive integer such that $\ell \gamma$ is a primitive element of the integer lattice $\mathbb{Z}^{m+1} \cong \mathbb{Z}_{\mathfrak{g}}^{*}$.

(2) $\left\langle\gamma, \lambda_{i}\right\rangle=-1$.

We say that a good rational polyhedral cone $C$ is associated with a toric diagram of height $\ell$ if there exists a rational vector $\gamma$ satisfying (1) and (2) above.

The reason why we use the terminology "height $\ell$ " is because of the following proposition.

Proposition 3.3. Using a transformation by an element of $S L(m+1, \mathbb{Z})$ we may assume that

$$
\gamma=\left(\begin{array}{r}
-\frac{1}{\ell} \\
0 \\
\vdots \\
0
\end{array}\right)
$$

and the first component of $\lambda_{i}$ is equal to $\ell$ for each $i$.

Proof. By elementary group theory there is an element $A$ of $S L(m+1, \mathbb{Z})$ which sends the primitive vector $\ell \gamma$ in $\mathbb{Z}^{m+1}$ to ${ }^{t}(-1,0, \cdots, 0)$ where the left upper $t$ denotes the transpose. Then $A \gamma={ }^{t}\left(-\frac{1}{\ell}, 0, \cdots, 0\right)$. By transforming $\mathfrak{g}$ by ${ }^{t} A^{-1}$, the transpose of $A^{-1}$, we get

$$
\left\langle A \gamma,{ }^{t} A^{-1} \lambda_{i}\right\rangle=\left\langle\gamma, \lambda_{i}\right\rangle=-1 \text {. }
$$

This implies the first component of ${ }^{t} A^{-1} \lambda_{i}$ is $\ell$.

Before we give a proof of Theorem 1.2 we outline the proof of the following fact (c.f. [16, 20]).

Proposition 3.4. For each pair of a good rational polyhedral cone $C$ and an element $\xi \in C_{0}^{*}$ where

$$
C_{0}^{*}=\{\xi \in \mathfrak{g} \mid\langle v, \xi\rangle>0 \text { for all } v \in C\}
$$

there is a compact connected toric Sasaki manifold $S$ whose moment map image is equal to $C \backslash\{0\}$ and whose Reeb vector field is generated by $\xi$.

Outline of the proof. The construction of a contact manifold from a good rational polyhedral cone is the so-called Delzant construction. Let $e_{1}, \cdots, e_{d}$ be the canonical basis of $\mathbb{R}^{d}$. Of course they generate the lattice $\mathbb{Z}^{d}$. Let $\beta_{\mathbb{Z}}: \mathbb{Z}^{d} \rightarrow \mathbb{Z}_{\mathfrak{g}} \cong \mathbb{Z}^{m+1}$ be the homomorphism defined by

$$
\beta_{\mathbb{Z}}\left(e_{i}\right)=\lambda_{i},
$$

and $\beta_{\mathbb{R}}: \mathbb{R}^{d} \rightarrow \mathfrak{g} \cong \mathbb{R}^{m+1}$ be the natural linear map induced by $\beta_{\mathbb{Z}}$. Since $C$ has non-empty interior then $\beta_{\mathbb{R}}$ is surjective, i.e. there is a subset $\left\{i_{1}, \cdots, i_{m+1}\right\}$ such that $\lambda_{i_{1}}, \cdots, \lambda_{i_{m+1}}$ are linearly independent over $\mathbb{R}$. Then $\beta_{\mathbb{Z}}$ and $\beta_{\mathbb{R}}$ naturally 
induce a homomorphism $\beta_{T}: T^{d} \rightarrow G \cong T^{m+1}$ of the tori. Let $K$ be the kernel of $\beta_{T}$. We write $[a] \in T^{d}$ for the image of $a \in \mathbb{R}^{d}$. Then

$$
K=\left\{[a] \mid \sum_{i=1}^{d} a_{i} \lambda_{i} \in \mathbb{Z}_{\mathfrak{g}}\right\} .
$$

It is a compact abelian subgroup of $T^{d}$ and its Lie algebra is ker $\beta_{\mathbb{R}}$. Note that $K$ is not connected in general. Let us consider the standard action of $T^{d}$ on $\mathbb{C}^{d}$ with the Kähler form $\frac{i}{2} \sum_{i=1}^{d} d v^{i} \wedge d \bar{v}^{j}$ by

$$
[a] \cdot\left(v^{1}, \cdots, v^{d}\right)=\left(e^{2 \pi i a_{1}} v^{1}, \cdots, e^{2 \pi i a_{d}} v^{d}\right) .
$$

Consider the action of $K$ on $\mathbb{C}^{d}$ obtained as the restriction of the $T^{d}$-action and the moment map $\mu_{K}: \mathbb{C}^{d} \rightarrow \mathfrak{k}^{*}$. The Kähler cone manifold $C(S)$ is obtained as the Kähler quotient

$$
C(S)=\left(\mu_{K}^{-1}(0) \backslash\{0\}\right) / K \text {. }
$$

See [16] for more detail. The closure $\overline{C(S)}$ is obtained as

$$
\overline{C(S)}=\mu_{K}^{-1}(0) / K
$$

which is realized also as a normal complex analytic space via the standard method using fans in algebraic geometry ([23]).

A Sasaki manifold $S$ is obtained as

$$
S=\left(\mu_{K}^{-1}(0) \cap S^{2 d-1}\right) / K
$$

where $S^{2 d-1}$ is the standard $(2 d-1)$-sphere in $\mathbb{C}^{d}$. This Sasaki metric is often called the canonical Sasaki metric, and the symplectic potential on $C(S)$ and the Reeb field are respectively given by

$$
\begin{aligned}
G^{c a n} & =\frac{1}{2} \sum_{i=1}^{d} l_{i}(y) \log l_{i}(y), \\
\xi^{c a n} & =\sum_{i=1}^{d} \lambda_{i}
\end{aligned}
$$

where $l_{i}(y)=\left\langle\lambda_{i}, y\right\rangle$. For a general Reeb field $\xi \in \mathfrak{g}$ a symplectic potential $G_{\xi}^{c a n}$ on $C(S)$ is given by

$$
G_{\xi}^{c a n}(y)=\frac{1}{2} \sum_{i=1}^{d} l_{i}(y) \log l_{i}(y)+\frac{1}{2} l_{\xi}(y) \log l_{\xi}(y)-\frac{1}{2} l_{\infty}(y) \log l_{\infty}(y)
$$

where $l_{\xi}(y)=\langle\xi, y\rangle$ and $l_{\infty}=\left\langle\xi^{c a n}, y\right\rangle$, see [20] for more detail. The corresponding Kähler potential $F_{\xi}^{c a n}$, computed by the Legendre transform, is given by

$$
F_{\xi}^{c a n}=\frac{1}{2} l_{\xi}(y),
$$

see (61) in [11]. Since the Kähler potential is equal to $\frac{1}{2} r^{2}$ then $r^{2}=l_{\xi}(y)$ and the Sasakian structure is determined via the identification $S \cong\left\{l_{\xi}(y)=1\right\} \subset C(S)$.

Proof of Theorem 1.2: First we prove that (a) implies (b). Suppose $c_{1}^{B}>0$ and $c_{1}(D)=0$. By our assumption $(m+1)$-dimensional torus $G$ acts on $S$ preserving 
the Sasakian structure. By Proposition 4.3 in [1] we can then choose a $G$-invariant transverse Kähler form $\omega^{T}$ such that

$$
c_{1}^{B}=(2 m+2)\left[\omega^{T}\right] .
$$

Let $\rho^{T}$ be the Ricci form of $\omega^{T}$. Note that $\omega^{T}$ and $\rho^{T}$ are defined on each local leaf spaces of the Reeb foliation, but they can be lifted to $S$ to define global 2-forms on $S$. There exists a basic $G$-invariant smooth function $h$ on $S$ such that

$$
\rho^{T}=(2 m+2) \omega^{T}+i \partial_{B} \bar{\partial}_{B} h
$$

on $S$. By an elementary curvature computation in Sasakian geometry the equation (13) is equivalent to

$$
\rho=-i \partial \bar{\partial} \log \operatorname{det}\left(F_{i j}\right)=i \partial \bar{\partial} h
$$

on $C(S)$ where $h$ is pulled back to $C(S) \cong \mathbb{R}_{+} \times S$ so that $h$ satisfies

$$
r \frac{r}{\partial r} h=\xi h=0
$$

and where $F$ is the Kähler potential on $C(S),\left(e^{x^{0}+i \theta^{0}}, \cdots, e^{x^{m}+i \theta^{m}}\right)$ is the coordinates of $G^{\mathbb{C}} \cong\left(\mathbb{C}^{*}\right)^{m+1}$ and

$$
F_{i j}=\frac{\partial^{2} F}{\partial x^{i} \partial x^{j}} .
$$

Note that since $F$ is $G$-invariant it is independent of $\theta^{i}$ 's. Since any $G$-invariant pluriharmonic function on $C(S)$ is an affine function then there exists a $\gamma \in \mathfrak{g}^{*}$ such that

$$
\log \operatorname{det}\left(F_{i j}\right)=-2 \sum_{i=0}^{m} \gamma_{i} x^{i}-h
$$

by replacing $h+$ constant by $h$. Using the Legendre transform $G$ of $F$ we get

$$
\log \operatorname{det}\left(G_{i j}\right)=2 \sum_{i=0}^{m} \gamma_{i} G_{i}+h .
$$

Using Abreu-Guillemin arguments about the boundary behavior of $G$ it is shown in 20] that

$$
\left\langle\lambda_{j}, \gamma\right\rangle=-1 \quad \text { for } j=1, \cdots, d .
$$

Since the moment map image has non-empty interior there are $(m+1)$ vectors $\lambda_{j_{1}}, \cdots, \lambda_{j_{m+1}}$ linearly independent over $\mathbb{R}$. Hence one can consider $\gamma$ as a solution to the linear equations

$$
\left\langle\lambda_{j_{i}}, \gamma\right\rangle=-1 \quad \text { for } i=1, \cdots, m+1
$$

and sees that $\gamma \in \mathbb{Q}_{\mathfrak{g}^{*}}^{m+1}$. Choosing a positive integer $\ell$ such that $\ell \gamma$ is a primitive element of the integer lattice. Since $\eta(\xi)=1$ and the moment map on $C(S)$ is given by $\frac{1}{2} r^{2} \eta$ we have $\langle y, \xi\rangle>0$ for all $y \in C$. It is also shown in [20] that $\langle\gamma, \xi\rangle=-m-1$. This proves that (a) implies (b).

Next we prove that (b) implies (c). Return to the Delzant construction in the proof of Proposition 3.4. One sees that $\mu_{K}^{-1}(0)$ is given by

$$
\mu_{K}^{-1}(0)=\left\{\left.v \in \mathbb{C}^{d}\left|\sum_{i=1}^{d} b_{i}\right| v_{i}\right|^{2}=0 \text { for all } b \in \mathfrak{k} \subset \mathbb{R}^{d}\right\} .
$$


By Proposition 3.3 we may assume that the first component of $\lambda_{i}$ is $\ell$ for each $i$. Recall that

$$
\sum_{i=1}^{d} a_{i} \lambda_{i} \in \mathbb{Z}^{m+1}
$$

for all $[a] \in K$. Looking at the first component we get

$$
\ell\left(a_{1}+\cdots+a_{d}\right) \in \mathbb{Z}
$$

for all $[a] \in K$. Thus

$$
\left(e^{2 \pi i\left(a_{1}+\cdots+a_{d}\right)} d v_{1} \wedge \cdots \wedge d v_{d}\right)^{\otimes \ell}=\left(d v_{1} \wedge \cdots \wedge d v_{d}\right)^{\otimes \ell} .
$$

Let $\mathbf{b}_{1}, \cdots, \mathbf{b}_{d-m-1}$ be a basis of $\mathfrak{k}$, and put $\mathbf{b}_{i}=\left(b_{i 1}, \cdots, b_{i d}\right)$ and

$$
X_{i}=\sum_{j=1}^{d} b_{i j} \frac{\partial}{\partial v^{j}} .
$$

Then

$$
\left(i\left(X_{1}\right) \cdots i\left(X_{d-m-1}\right) d v_{1} \wedge \cdots \wedge d v_{d}\right)^{\otimes \ell}
$$

descends to a nowhere zero section of $K_{C(S)}^{\otimes \ell}$. Hence $K_{C(S)}^{\otimes \ell}$ is a trivial line bundle. This proves that (d) implies (c). Note that this proof shows the section (19) extends to the apex of $\overline{C(S)}$, as a token of $\mathbb{Q}$-Gorenstein property (c.f. Remark 1.3 ).

We now prove that (c) implies (a). Suppose we are given a $G$-invariant Sasakian structure with Reeb field $\xi$ and with trivial line bundle $K_{C(S)}^{\otimes \ell}$. Thus we have a $G$-invariant Kähler metric $\omega$ and a nowhere vanishing holomorphic section $\Omega_{1}$ of $K_{C(S)}^{\otimes \ell}$. Let $h_{1}$ be defined by

$$
h_{1}=\frac{1}{\ell} \log \left\|\Omega_{1}\right\|^{2}
$$

where the norm of $\Omega_{1}$ is taken with respect to $\omega$. Then the Ricci form $\rho$ of $\omega$ is written as

$$
\rho=\frac{1}{2 \pi} \partial \bar{\partial} h_{1}
$$

Let $h$ be the average of $h_{1}$ by the action of $G$. Since $\rho$ is $G$-invariant we see from (20) that

$$
\rho=\frac{i}{2 \pi} \partial \bar{\partial} h
$$

Starting from (14) which is identical to (21) we get (16) and (17) (though we do not have (15)). Then it is shown in 20] that

$$
\langle\xi, \gamma\rangle=-(m+1) .
$$

The equation (16) says that $e^{h} \operatorname{det}\left(F_{i j}\right)$ is a flat metric on $C(S)$. Consider the $(m+1)$-form $\Omega$ written as

$$
\begin{aligned}
\Omega & =e^{-i \sum_{i=0}^{m} \gamma_{i} \theta^{i}} e^{\frac{h}{2}}\left(\operatorname{det}\left(F_{i j}\right)\right)^{\frac{1}{2}} d z^{0} \wedge \cdots \wedge d z^{m} \\
& =e^{-\sum_{i=0}^{m} \gamma_{i} z^{i}} d z^{0} \wedge \cdots \wedge d z^{m}
\end{aligned}
$$

where we used (16). Then $\Omega$ is multi-valued if $\gamma$ is not integral but only rational. Let $\ell_{1}$ be the positive integer such that $\ell_{1} \gamma$ is a primitive element of the integer lattice. Then $\Omega^{\otimes \ell_{1}}$ is a holomorphic section of $K_{C(S}^{\otimes \ell_{1}}$ over the open set corresponding 
to the interior of the moment map image. But since $\left\|\Omega^{\otimes \ell_{1}}\right\|=1$ we see that $\Omega^{\otimes \ell_{1}}$ extends to the whole $C(S)$. We further have

$$
\mathcal{L}_{\xi} \Omega=(m+1) i \Omega
$$

and

$$
\left(\frac{i}{2}\right)^{m+1}(-1)^{m(m+1) / 2} \Omega \wedge \bar{\Omega}=\exp (h) \frac{1}{(m+1) !} \omega^{m+1} .
$$

Since $\xi$ is decomposed into the holomorphic and the anti-holomorphic parts

$$
\xi=\frac{1}{2}(\xi-i J \xi)+\frac{1}{2}(\xi+i J \xi)
$$

with $J \xi=-r \frac{\partial}{\partial r}$ we have

$$
\begin{aligned}
\mathcal{L}_{\xi} \Omega & =\mathcal{L}_{\frac{1}{2}\left(\xi+i r \frac{\partial}{\partial r}\right)} \Omega \\
& =\frac{(m+1) i}{2} \Omega+\frac{i}{2} \mathcal{L}_{r \frac{\partial}{\partial r}} \Omega .
\end{aligned}
$$

From this and (24) it follows that

$$
\mathcal{L}_{r \frac{\partial}{\partial r}} \Omega=(m+1) \Omega
$$

and

$$
\mathcal{L}_{r \frac{\partial}{\partial r}}(\Omega \wedge \bar{\Omega})=2(m+1) \Omega \wedge \bar{\Omega} .
$$

On the other hand since $\omega=i \partial \bar{\partial}\left(r^{2} / 2\right)$ we have

$$
\mathcal{L}_{r \frac{\partial}{\partial r}} \omega^{m+1}=2(m+1) \omega^{m+1} .
$$

Taking the Lie derivative of both sides of (25) by $r \frac{\partial}{\partial r}$ we get

$$
r \frac{\partial h}{\partial r}=0 .
$$

Since $h$ is $G$-invariant we also have $\xi h=0$. Hence (25) implies $\left[\rho^{T}\right]=(2 m+$ 2) $\left[\omega^{T}\right]$ as basic cohomology classes, from which we get $c_{1}^{B}>0$ and $c_{1}(D)=0$ by Proposition 4.3 in [11. This completes the proof of Theorem 1.2 .

\section{EXAMPLES AND REMARKS ON THE FUNDAMENTAL GROUPS}

As is mentioned in the introduction there are examples of 3 dimensional toric diagrams of height 1 denoted by $X^{p, q}, Y^{p, q}, Z^{p, q}$ and $L^{p, q, r}$ known in physics literature and all the corresponding Sasaki manifolds have Sasaki-Einstein metrics by the existence result of [11. To check that these toric diagrams satisfy the goodness condition of Definition 3.2 the following proposition is useful.

Proposition 4.1. Let $C$ be a convex polyhedral cone in $\mathbb{R}^{3}$ given by

$$
C=\left\{y \in \mathbb{R}^{3} \mid\left\langle y, \lambda_{i}\right\rangle \geq 0, j=1, \cdots, d\right\}
$$

with

$$
\lambda_{1}=\left(\begin{array}{c}
1 \\
p_{1} \\
q_{1}
\end{array}\right), \cdots, \lambda_{d}=\left(\begin{array}{c}
1 \\
p_{d} \\
q_{d}
\end{array}\right) .
$$

Then $C$ is good in the sense of Definition 3.2 if and only if either

(i) $\left|p_{i+1}-p_{i}\right|=1$ or $\left|q_{i+1}-q_{i}\right|=1$

or

(ii) $p_{i+1}-p_{i}$ and $q_{i+1}-q_{i}$ are relatively prime non-zero integers 
for $i=1, \cdots, d$ where we have put $\lambda_{d+1}=\lambda_{1}$.

Further the area of the 2-dimensional convex polytope formed by

$$
\left(\begin{array}{c}
p_{1} \\
q_{1}
\end{array}\right), \cdots,\left(\begin{array}{c}
p_{d} \\
q_{d}
\end{array}\right),\left(\begin{array}{c}
p_{1} \\
q_{1}
\end{array}\right)
$$

is an invariant of the equivalent classes given by the action of some element of $S L(3, \mathbb{Z})$ on the set of all such diagrams.

Proof. Let $a_{1}$ and $a_{2}$ be real numbers such that $a_{1} \lambda_{i}+a_{2} \lambda_{i+1} \in \mathbb{Z}^{3}$. Then we have

$$
a_{1}+a_{2} \in \mathbb{Z}, p_{i} a_{1}+p_{i+1} a_{2} \in \mathbb{Z}, q_{i} a_{1}+q_{i+1} a_{2} \in \mathbb{Z} .
$$

It follows from these that

$$
\left(p_{i+1}-p_{i}\right) a_{2} \in \mathbb{Z},\left(q_{i+1}-q_{i}\right) a_{2} \in \mathbb{Z} .
$$

If $\left(p_{i+1}-p_{i}\right)$ and $\left(q_{i+1}-q_{i}\right)$ satisfy (i) or (ii) then there exist $s, t \in \mathbb{Z}$ such that $s\left(p_{i+1}-p_{i}\right)+t\left(q_{i+1}-q_{i}\right)=1$. Then from (27) we get $a_{2} \in \mathbb{Z}$. From (26) we also have $a_{1} \in \mathbb{Z}$. Conversely if $a_{2}$ satisfying (27) is always in $\mathbb{Z}$ then $\left(p_{i+1}-p_{i}\right)$ and $\left(q_{i+1}-q_{i}\right)$ are relatively prime.

If a diagram of the first variable 1 is transformed to another by an element of $S L(3, \mathbb{Z})$ then the volume of the 3 -dimensional truncated cone

$$
\left\{a_{1} \lambda_{1}+\cdots+a_{d} \lambda_{d} \mid 0 \leq a_{1} \leq 1, \cdots, 0 \leq a_{d} \leq 1,0 \leq a_{1}+\cdots+a_{d} \leq 1\right\}
$$

is invariant. But this is equal to one thirds of the area described in the statement of the proposition. This completes the proof of Proposition 4.1.

We give a simplest toric diagram of hight $\ell$. Let $C$ be the convex polyhedral cone defined by

$$
C=\left\{y \in \mathbb{R}^{3} \mid\left\langle y, \lambda_{i}\right\rangle \geq 0, j=1,2,3\right\}
$$

with

$$
\lambda_{1}=\left(\begin{array}{l}
1 \\
0 \\
0
\end{array}\right), \lambda_{2}=\left(\begin{array}{l}
0 \\
1 \\
0
\end{array}\right), \lambda_{3}=\left(\begin{array}{l}
1 \\
1 \\
\ell
\end{array}\right) .
$$

Then this is a good cone and defines a smooth Sasaki manifold. One can show that

$$
\gamma=\left(\begin{array}{c}
-1 \\
-1 \\
\frac{1}{\ell}
\end{array}\right)
$$

Taking

$$
A=\left(\begin{array}{rrr}
0 & 0 & -1 \\
-1 & 1 & 0 \\
1 & 0 & \ell
\end{array}\right)
$$

we have

$$
A \gamma=\left(\begin{array}{c}
-\frac{1}{\ell} \\
0 \\
0
\end{array}\right),{ }^{t} A^{-1} \lambda_{1}=\left(\begin{array}{c}
\ell \\
0 \\
1
\end{array}\right),{ }^{t} A^{-1} \lambda_{2}=\left(\begin{array}{c}
\ell \\
1 \\
1
\end{array}\right),{ }^{t} A^{-1} \lambda_{3}=\left(\begin{array}{c}
\ell \\
1 \\
2
\end{array}\right) .
$$

By following the Delzant construction one sees that the resulting Sasaki manifold is the Lens space $S^{5} / \mathbb{Z}_{\ell}$.

Next let $C$ be the convex polyhedral cone defined by

$$
C=\left\{y \in \mathbb{R}^{3} \mid\left\langle y, \lambda_{i}\right\rangle \geq 0, j=1,2,3,4\right\}
$$


with

$$
\lambda_{1}=\left(\begin{array}{l}
1 \\
0 \\
0
\end{array}\right), \lambda_{2}=\left(\begin{array}{l}
0 \\
1 \\
0
\end{array}\right), \lambda_{3}=\left(\begin{array}{l}
1 \\
1 \\
\ell
\end{array}\right), \lambda_{4}=\left(\begin{array}{c}
1 \\
1 \\
\ell-1
\end{array}\right) .
$$

Then this is a good cone and defines a smooth Sasaki manifold. The resulting Sasaki manifold does not satisfy the conditions of Theorem 1.2 because there is no $\gamma$ with $\left\langle\gamma, \lambda_{j}\right\rangle=-1$ for $j=1,2,3,4$.

One can show that if $\ell>1$ then the resulting Sasaki manifold $S$ is not simply connected. This follows from a result of Lerman [17] which is stated as follows. Let $\mathcal{L}$ be the subgroup of $\mathbb{Z}_{\mathfrak{g}}$ generated by $\lambda_{1}, \cdots, \lambda_{d}$. Then $\pi_{1}(S) \cong \mathbb{Z}_{\mathfrak{g}} / \mathcal{L}$. Obviously $\mathbb{Z}_{\mathfrak{g}} / \mathcal{L}$ is not trivial if $\ell>1$. Thus we proved the following.

Proposition 4.2. Let $S$ be a compact connected toric Sasaki manifold associated with a toric diagram of height $\ell>1$. Then $S$ is not simply connected.

Note that the converse is not true as the following example shows. Consider the toric diagram with height 1 defined by the three normal vectors

$$
\lambda_{1}=\left(\begin{array}{l}
1 \\
0 \\
0
\end{array}\right), \lambda_{2}=\left(\begin{array}{l}
1 \\
2 \\
1
\end{array}\right), \lambda_{3}=\left(\begin{array}{l}
1 \\
3 \\
4
\end{array}\right) .
$$

The resulting Sasaki manifold is the Lense space with a different $\mathbb{Z}_{5}$-action from the above example with $\ell=5$. Note also for example $Y^{p, q}$ is not simply connected unless $p$ and $q$ are relatively prime.

Proof of Theorem 1.5: Put $n=k+3$. We construct diagrams of height 1 with either $\left|p_{i+1}-p_{i}\right|=1$ or $\left|q_{i+1}-q_{i}\right|=1$ such that $\left(p_{1}, q_{1}\right), \cdots,\left(p_{n}, q_{n}\right),\left(p_{1}, q_{1}\right)$ form a convex polytope with $n$ vertices and that they generate $\mathbb{Z}^{2}$. Then $S$ is simply connected since $\mathcal{L}=\mathbb{Z}^{3}$. By another theorem of Lerman [17] we know that $b_{2}(S)=n-3=k$. It follows from the classification of five dimensional simply connected spin manifolds with $T^{3}$-action ([6]) that $S=S^{5} \sharp k\left(S^{2} \times S^{5}\right)$.

There are many ways to construct such examples. For instance if $k=2 r$ so that $n=2 r+3$ then take

$$
\begin{gathered}
\left(\begin{array}{c}
p_{0} \\
q_{0}
\end{array}\right)=\left(\begin{array}{l}
0 \\
0
\end{array}\right),\left(\begin{array}{c}
p_{1} \\
q_{1}
\end{array}\right)=\left(\begin{array}{c}
1 \\
1
\end{array}\right), \\
\ldots,\left(\begin{array}{c}
p_{r} \\
q_{r}
\end{array}\right)=\left(\begin{array}{c}
r \\
\frac{r(r+1)}{2}
\end{array}\right),\left(\begin{array}{c}
p_{r+1} \\
q_{r+1}
\end{array}\right)=\left(\begin{array}{c}
r+1 \\
\frac{(r+1)(r+2)}{2}+s
\end{array}\right), \\
\left(\begin{array}{c}
p_{r+2} \\
q_{r+2}
\end{array}\right)=\left(\begin{array}{c}
r \\
\frac{(r+1)(r+2)}{2}+s-1
\end{array}\right), \cdots, \\
\left(\begin{array}{c}
p_{2 r+1} \\
q_{2 r+1}
\end{array}\right)=\left(\begin{array}{c}
p_{2 r+2} \\
\frac{(r+1)(r+2)}{2}+s-\frac{r(r+1)}{2}
\end{array}\right),\left(\begin{array}{l}
0 \\
q_{2 r+2}
\end{array}\right) .
\end{gathered}
$$

For different values of $s$ they give inequivalent toric diagrams because they have different areas. If $k=2 r-1$ so that $n=2 r+2$ then take

$$
\begin{gathered}
\left(\begin{array}{c}
p_{0} \\
q_{0}
\end{array}\right)=\left(\begin{array}{l}
0 \\
0
\end{array}\right),\left(\begin{array}{c}
p_{1} \\
q_{1}
\end{array}\right)=\left(\begin{array}{c}
1 \\
1
\end{array}\right),\left(\begin{array}{l}
p_{2} \\
q_{2}
\end{array}\right)=\left(\begin{array}{l}
2 \\
3
\end{array}\right), \\
\cdots,\left(\begin{array}{c}
p_{r-1} \\
q_{r-1}
\end{array}\right)=\left(\begin{array}{c}
r-1 \\
\frac{(r-1) r}{2}
\end{array}\right)
\end{gathered}
$$




$$
\begin{gathered}
\left(\begin{array}{c}
p_{r} \\
q_{r}
\end{array}\right)=\left(\begin{array}{c}
r \\
\frac{r(r+1)}{2}+s
\end{array}\right),\left(\begin{array}{l}
p_{r+1} \\
q_{r+1}
\end{array}\right)=\left(\begin{array}{c}
0 \\
\frac{(r)(r+1)}{2}+s+1
\end{array}\right), \\
\left(\begin{array}{c}
p_{r+3} \\
q_{r+3}
\end{array}\right)=\left(\begin{array}{c}
-r \\
\frac{r(r+1)}{2}+s
\end{array}\right),\left(\begin{array}{c}
p_{r+4} \\
q_{r+4}
\end{array}\right)=\left(\begin{array}{c}
-(r-1) \\
\frac{(r-1) r}{2}
\end{array}\right), \\
\ldots,\left(\begin{array}{c}
p_{2 r} \\
q_{2 r}
\end{array}\right)=\left(\begin{array}{c}
-2 \\
3
\end{array}\right)\left(\begin{array}{c}
p_{2 r+1} \\
q_{2 r+1}
\end{array}\right)=\left(\begin{array}{c}
-1 \\
0
\end{array}\right) .
\end{gathered}
$$

Then again different values of $s$ give inequivalent diagrams. This completes the proof of Theorem 1.5 .

\section{REFERENCES}

[1] M. Abreu : Kähler geometry of toric manifolds in symplectic coordinates, Fields Institute Comm. vol. 35, 1-24, AMS, 2003.

[2] K. Altman : Toric $\mathbb{Q}$-Gorenstein singularities, ArXiv:alg-geom/9403003

[3] R. Argurio, M. Bertolini, C. Closset and S. Cremonesi,On Stable Non-Supersymmetric Vacua at the Bottom of Cascading Theories, arXiv:hep-th/0606175.

[4] C. P. Boyer, K. Galicki, and J. Kollár, Einstein metrics on spheres, Ann. of Math.,162 (2005), no. $1,557-580$.

[5] C. P. Boyer, K. Galicki, and M. Nakamaye : On the geometry of Sasakian-Einstein 5manifolds, Math. Ann. 325 (2003), no. 3, 485-524.

[6] C. P. Boyer, K. Galicki and L. Ornea : Constructions in Sasakian geometry, math.DG/0602233

[7] C.P. Boyer, K. Galicki and S.R. Simanca, Canonical Sasakian metrics, preprint, math.DG/0604325

[8] X.X. Chen : The space of Kähler metrics, J. Diff. Geom. 56(2000), 189-234.

[9] M. Cvetič, H. Lü, D. N. Page and C. N. Pope, New Einstein-Sasaki Spaces in Five and Higher Dimensions, Phys. Rev. Lett. 95 (2005) 071101 (hep-th/0504225).

[10] S.K. Donaldson : Symmetric spaces, Kähler geometry and Hamiltonian dynamics, in Northern California Symplectic Geometry Seminar' (Eliashberg et al eds.), Amer. Math. Soc. 1999, 13-33.

[11] A. Futaki, H. Ono and G. Wang : Transverse Kähler geometry of Sasaki manifolds and toric Sasaki-Einstein manifolds, math.DG/0607586

[12] J. P. Gauntlett, D. Martelli, J. Sparks and D. Waldram, Sasaki-Einstein Metrics on $S^{2} \times S^{3}$, Adv. Theor. Math. Phys. 8 (2004) 711-734 (hep-th/0403002).

[13] D. Guan : On modified Mabuchi functional and Mabuchi moduli space of Kähler metrics on toric bundles, Math. Res. Letters, 6 (1999), 547-555.

[14] A. Hanany, P. Kazakopoulos and B. Wecht, A New Infinite Class of Quiver Gauge Theories, hep-th/0503177

[15] J. Kollár, Einstein metrics on connected sums of $S^{2} \times S^{3}$, math.DG/0402141 (2004).

[16] E. Lerman : Contact toric manifolds, J. Symplectic Geom. 1 (2003), no. 4, 785-828.

[17] E. Lerman : Homotopy groups of K-contact toric manifolds, Transact. Amer. Math. Soc., 356 (2004), no. 4, 4075-4084.

[18] T. Mabuchi : Some Sympletic geometry on compact Kähler manifolds. I, Osaka J. Math. 24 (1987), 227-252.

[19] D. Martelli and J. Sparks, Toric Sasaki-Einstein metrics on $S^{2} \times S^{3}$, Phys. Lett. B $621(2005)$ 208-212 (hep-th/0505027).

[20] D. Martelli, J. Sparks and S.-T. Yau : The geometric dual of a-maximisation for toric SasakiEinstein manifolds, Commun. Math. Phys. 268, 39-65 (2006). (hep-th/0503183).

[21] D. Martelli, J. Sparks and S.-T. Yau : Sasaki-Einstein manifolds and volume minimisation, arXiv:hep-th/0603021.

[22] S. Nishikawa and P. Tondeur : Transversal infinitesimal automorphisms for harmonic Kähler foliation, Tohoku Math. J., 40(1988), 599-611. 
[23] T. Oda : Convex bodies and algebraic geometry - An introduction to the theory of toric varieties. Ergebnisse der Mathematik und ihrer Grenzgebiete 15. Berlin: Springer-Verlag, 1988.

[24] T. Oota and Y. Yasui : New Example of Infinite Family of Quiver Gauge Theories, arXiv:hep-th/0610092

[25] S. Semmes : Complex Monge-Ampère and symplectic manifolds, Amer. J. Math., 114(1992), 495-550.

[26] C. van Coevering : Toric surfaces and Sasakian-Einstein 5-manifolds, math.DG/0607721

Department of Mathematics, Kyushu University, 6-10-1, Hakozaki, Higashiku, FukuokaCITY, FUKUOKA 812-8581 JAPAN

E-mail address: cho@math.kyushu-u.ac.jp

Department of Mathematics, Tokyo Institute of Technology, 2-12-1, O-okayama, Meguro, TOKYO 152-8551, JaPAN

E-mail address: futaki@math.titech.ac.jp

Department of Mathematics, Tokyo Institute of Technology, 2-12-1, O-okayama, Meguro, TOKYO 152-8551, Japan

E-mail address: ono@math.titech.ac.jp 